\title{
COMPACT HOMOGENEOUS HYPERSURFACES
}

\author{
BY \\ SHOSHICHI KOBAYASHI
}

The purpose of the present paper is to prove the following

Theorem. If an n-dimensional compact homogeneous Riemannian space $M$ can be imbedded isometrically into the $(n+1)$-dimensional Euclidean space $R^{n+1}$, then $M$ is isometric to a sphere.

By "homogeneous" we mean that the group $I(M)$ of isometries of $M$ is transitive on $M$. By "imbedding" we mean a locally one-to-one mapping of $M$ into $R^{n+1}$ of class $C^{2}$.

The outline of the proof is as follows. (1) Since $M$ is compact, there exists a point of $M$ in a neighborhood of which $M$ is locally convex. (2) This neighborhood is rigid if $n \geqq 3$. (3) Using the homogeneity of $M$ and the rigidity of the above neighborhood, we show that $M$ is orientable and rigid. (4) Furthermore, the spherical map of Gauss is a homeomorphism of $M$ onto the unit sphere. (5) Since $M$ is rigid, every isometry is the restriction of a motion in $R^{n+1}$ to $M$. (6) Therefore $I(M)$ can be considered as a subgroup of the group of motions in $R^{n+1}$. Since $I(M)$ is compact, it has a fixed point in $R^{n+1}$. (7) $M$ is a sphere with its center at this fixed point. (8) The case $n=2$ is treated at the end of the paper.

In the last section we indicate other proofs of the theorem which makes use of the theory of convex hypersurfaces. For these alternative proofs, the author owes many suggestions to Professors H. Busemann and S. S. Chern.

In the first two sections, we give, for the sake of completeness, a proof of the classical rigidity theorem which plays an important role in this paper. Although we make use of the connection theory in fibre bundles, our proof of the rigidity theorem is not new but due to E. Cartan.

\section{Preliminary propositions.}

Notations. We denote by $R^{n}$ the $n$-dimensional real vector space (with or without a positive definite inner product) or the $n$-dimensional Euclidean space. If $M$ is a manifold $T_{x}(M)$ denotes the tangent space to $M$ at a point $x$. Let $f$ be a mapping of a manifold $M^{\prime}$ into $M$. Then $\delta f: T\left(M^{\prime}\right) \rightarrow T(M)$ is the differential of $f$, where $T(M)=\mathrm{U}_{x} T_{x}(M)$. If $\omega$ is a differential form on $M$, then $f^{*}(\omega)$ is the differential form on $M^{\prime}$ induced from $\omega$ by $f$.

Proposition 1. Let $M$ be an $n$-dimensional manifold with an $R^{n}$-valued linear differential form $\omega$ which defines an absolute parallelism on $M$; that is, if $\bar{x} \in T_{x}(M)$ and $\omega(\bar{x})=0$, then $\bar{x}$ is the zero vector at $x$. Let $f$ and $g$ be mappings of an $n^{\prime}$-dimensional manifold $M^{\prime}$ into $M$ such that

Received by the editors June 3, 1956 and, in revised form, November 14, 1956. 


$$
\begin{aligned}
& f\left(y_{0}\right)=g\left(y_{0}\right) \quad \text { for some point } y_{0} \text { in } M^{\prime} . \\
& f^{*}(\omega)=g^{*}(\omega) .
\end{aligned}
$$

Then $f(y)=g(y)$ for all $y$ in $M^{\prime}$.

Proof. Let $y$ be any point of $M^{\prime}$ and let $c$ be a differentiable curve from $y_{0}$ to $y$ with parameter $0 \leqq t \leqq 1$. Let $\bar{c}(t)$ be the vector tangent to $c$ at $c(t)$. Then

$$
\left\langle f^{*}(\omega), \bar{c}(t)\right\rangle=\langle\omega, \delta f(\bar{c}(t))\rangle, \quad\left\langle g^{*}(\omega), \bar{c}(t)\right\rangle=\langle\omega, \delta g(\bar{c}(t))\rangle .
$$

From the assumption (2) we obtain

$$
\langle\omega, \delta f(\bar{c}(t))\rangle=\langle\omega, \delta g(\bar{c}(t))\rangle, \quad 0 \leqq t \leqq 1 .
$$

Put

$$
c_{1}=f(c), \quad c_{2}=g(c) .
$$

Let $\bar{c}_{1}(t)$ (resp. $\left.\bar{c}_{2}(t)\right)$ be the vector tangent to $c_{1}\left(\right.$ resp. $\left.c_{2}\right)$ at $c_{1}(t)\left(\right.$ resp. $\left.c_{2}(t)\right)$. Then

$$
\bar{c}_{1}(t)=\delta f(\bar{c}(t)), \quad \bar{c}_{2}(t)=\delta g(\bar{c}(t)) .
$$

Therefore we have

$$
c_{1}(0)=c_{2}(0) \text { and }\left\langle\omega, \bar{c}_{1}(t)\right\rangle=\left\langle\omega, \bar{c}_{2}(t)\right\rangle \quad 0 \leqq t \leqq 1 .
$$

Since $\omega$ defines an absolute parallelism, it follows (from the uniqueness theorem on linear differential equations) that

$$
c_{1}(t)=c_{2}(t) \quad 0 \leqq t \leqq 1 .
$$

Hence

$$
f(y)=g(y)
$$

Let $M$ be an $n$-dimensional orientable Riemannian space isometrically imbedded in the Euclidean space $R^{n+1}$. Let $v(x)$ be one of two unit vectors perpendicular to $M$ at $x$. We choose $v(x)$ so that $v$ be continuous on $M$. This is possible because $M$ is orientable. Let $o$ be the origin of $R^{n+1}$ and let $S$ be the unit sphere in $R^{n+1}$ with its center at $o$. Let $\xi(x)$ be the point of $S$ such that the vector from $o$ to $\xi(x)$ is parallel to $v(x)$. Thus we obtain a mapping $\xi$ of $M$ into $S$, which is called the spherical map of Gauss.

Let $P$ (resp. $Q$ ) be the bundle of oriented orthonormal frames over $M$ (resp. $S$ ). Then $P$ (resp. $Q$ ) is a principal fibre bundle over $M$ (resp. $S$ ) with group $S O(n)$. Given an orthonormal frame $u$ on $M$ at $x$, we obtain, by parallel displacement in $R^{n+1}$, an orthonormal frame $u^{\prime}$ on $S$ at $\xi(x)$. If we choose a proper orientation on $S$, we obtain a bundle map $\bar{\xi}: P \rightarrow Q$ given by $\bar{\xi}(u)=u^{\prime}$.

Proposition 2. Let $M$ be an n-dimensional orientable Riemannian space and let $f: M \rightarrow R^{n+1}$ and $g: M \rightarrow R^{n+1}$ be two isometrical imbeddings of $M$ such that 
(1) $f\left(x_{0}\right)=g\left(x_{0}\right)$ for some point $x_{0}$ of $M$.

(2) The bundle map $\bar{\xi}$ above defined with respect to the imbedding $f$ is the same as the bundle map $\bar{\eta}$ with respect to the imbedding $g$. Then $f(x)=g(x)$ for all $x$ in $M$.

Proof. Let $M^{\prime}$ be the set of all points $x$ in $M$ such that $f(x)=g(x)$. Evidently $M^{\prime}$ is a nonempty closed subset of $M$. We shall show that $M^{\prime}$ is open in $M$. Let $x^{*}$ be any point of $M^{\prime}$ and let $x^{1}, \cdots, x^{n}$ be a local coordinate system in a neighborhood $U$ of $x^{*}$. Since $\bar{\xi}(x)=\bar{\eta}(x)$ for all $x$ in $M$, it follows that

$$
\partial f / \partial x^{i}=\partial g / \partial x^{i} \quad(i=1, \cdots, n) \text { at every point of } U .
$$

Since $f\left(x^{*}\right)=g\left(x^{*}\right)$, it follows that $f(x)=g(x)$ for all $x$ in $U$, thus proving Proposition 2.

2. Rigidity. Let $M$ be an $n$-dimensional orientable Riemannian space and let $P$ be the bundle of oriented orthonormal frames over $M$. We recall the definition of the form of soudure (soldering) [9]. It is an $R^{n}$-valued linear differential form $\theta$ defined on $P$ as follows. Let $\bar{u}$ be any vector tangent to $P$ at $u$ and let $\bar{x}$ be the projected image of $\bar{u}$ onto $M$. Since a frame $u \in P$ is an orthogonal transformation of $R^{n}$ onto $T_{x}(M), u^{-1}(\bar{x})$ is an element of $R^{n}$. We define

$$
\theta(\bar{u})=u^{-1}(\bar{x}) .
$$

The definition of $\theta$ in terms of local coordinate system is given in $[2 ; 8]$.

Let $s o(n)$ be the Lie algebra of $S O(n)$ and let $\omega$ be an $s o(n)$-valued linear differential form on $P$ defining a connection in $P$. Let $e_{1}, \cdots, e_{n}$ be an orthonormal basis for $R^{n}$. With respect to this basis, the Lie algebra $s o(n)$ can be considered as the Lie algebra of all skew-symmetric $(n-n)$-type matrices. Then $\theta$ is considered as a set of $n$ linear differential forms $\left(\theta^{\alpha}\right)_{\alpha=1}, \cdots, n$ and $\omega$ is a skew-symmetric matrix differential form $\left(\omega_{\beta}^{\alpha}\right)_{\alpha, \beta=1, \cdots, n}$. If

$$
d \theta^{\alpha}=-\sum \underset{\omega_{\beta}^{\alpha}}{{ }^{\alpha}} \wedge \theta^{\beta},
$$

then we say that $\omega$ defines the Riemannian connection. It is known that there exists a unique Riemannian connection.

Suppose that $M$ is imbedded isometrically into the $(n+1)$-dimensional Euclidean space $R^{n+1}$. Let $S$ be the unit sphere in $R^{n+1}$ and let $Q$ be the bundle of oriented orthonormal frames over $S$.

Let $\bar{\theta}$ be the form of soudure of the sphere $S$ and let $\bar{\omega}$ be the $s o(n)$-valued form on $Q$ defining the Riemannian connection on $S$. We proved in [9] that

$$
\omega=\bar{\xi}^{*}(\bar{\omega}) .
$$

It should be noted that $\bar{\xi}^{*}(\bar{\theta})$ is not equal to $\theta$ in general. We shall study the relation between $\bar{\xi}^{*}(\bar{\theta})$ and $\theta$. Put 


$$
\phi=\bar{\xi}^{*}(\bar{\theta}) .
$$

Define $\bar{\theta}^{\alpha}, \bar{\omega}_{\beta}^{\alpha}$ and $\phi^{\alpha}$ as before. Then we have

$$
\begin{aligned}
d \bar{\theta}^{\alpha} & =-\sum \bar{\omega}_{\beta}^{\alpha} \wedge \bar{\theta}^{\beta}, \\
d \bar{\omega}_{\beta}^{\alpha} & =-\sum \bar{\omega}_{\gamma}^{\alpha} \wedge \bar{\omega}_{\beta}^{\gamma}+\bar{\theta}^{\alpha} \wedge \bar{\theta}^{3}, \\
d \theta^{\alpha} & =-\sum \omega_{\beta}^{\alpha} \wedge \theta^{\beta}, \\
d \omega_{\beta}^{\alpha} & =-\sum \omega_{\gamma}^{\alpha} \wedge \omega_{\beta}^{\gamma}+\phi^{\alpha} \wedge \phi^{\beta} .
\end{aligned}
$$

Note that (d) follows immediately from (b).

REMARK. In the definition of the spherical map, we have two choices. If $\xi: M \rightarrow S$ is the spherical map associated with the orientation of $M$, then the other spherical map $\xi^{\prime}$ (associated with the reversed orientation of $M$ ) is given by $\xi^{\prime}=\sigma \circ \xi$, where is the symmetry of $S$ with respect to the center. It can be easily proved that

$$
-\bar{\xi}^{*}(\bar{\theta})=\bar{\xi}^{*}(\bar{\theta}),
$$

where $\bar{\xi}^{\prime}$ is defined similarly as $\bar{\xi}$. The form $\phi$ is a linear mapping of $T_{u}(P)$ into $R^{n}$ for each $u$ in $P$. The dimension of $\phi\left(T_{u}(P)\right)$ is called the type number at $u$ of the imbedded manifold $M$, denoted by $N(u)$.

Consider another isometrical imbedding of $M$ into $R^{n+1}$. Then we get another bundle map $\bar{\eta}: P \rightarrow Q$. Define $\psi=\bar{\eta}^{*}(\theta)$. Then

$$
d \omega_{\beta}^{\alpha}=-\sum \omega_{\gamma}^{\alpha} \wedge \omega_{\beta}^{\gamma}+\psi^{\alpha} \wedge \psi^{\beta} .
$$

Proposition 3. Consider two isometrical imbeddings of $M$ into $R^{n+1}$ and let $\bar{\xi}, \bar{\eta}: P \rightarrow Q$ be the bundle maps associated with them. We define $\phi$ and $\psi$ as above. If $N(u)=\operatorname{dim} \phi\left(T_{u}(P)\right) \geqq 3$, then $\phi= \pm \psi$ at $u$.

Proof. From (d) and (e), it follows that

$$
\phi^{\alpha} \wedge \phi^{\beta}=\psi^{\alpha} \wedge \psi^{\beta} \quad \alpha, \beta=1, \cdots, n .
$$

In particular

$$
\phi^{\alpha} \wedge \phi^{1} \wedge \psi^{1}=\psi^{\alpha} \wedge \psi^{1} \wedge \psi^{1}=0 \quad \alpha=1, \cdots, n .
$$

If $\phi^{1}$ and $\psi^{1}$ were linearly independent at $u$, then $\phi^{\alpha}$ would be a linear combination of $\phi^{1}$ and $\psi^{1}$ at $u$ for $\alpha=1, \cdots, n$, which contradicts to the assumption that $N(u) \geqq 3$. If $\psi^{1}$ vanishes at $u$, so does $\phi^{1}$; otherwise, from

$$
0=\psi^{\alpha} \wedge \psi^{1}=\phi^{\alpha} \wedge \phi^{1} \quad \text { at } u,
$$

we can conclude that $\phi^{\alpha}$ is proportional to $\phi^{1}$ at $u$ for every $\alpha$, which contradicts to the assumption that $N(u) \geqq 3$. Therefore there is a constant $\epsilon_{1}$ such that 


$$
\phi^{1}=\epsilon_{1} \psi^{1} \quad \text { at } u \text {. }
$$

Similarly, there exist $\epsilon_{2}, \cdots, \epsilon_{n}$ such that

$$
\phi^{2}=\epsilon_{2} \psi^{2}, \cdots, \phi^{n}=\epsilon_{n} \psi^{n} .
$$

From

$$
\phi^{1} \wedge \phi^{2}=\psi^{1} \wedge \psi^{2}, \quad \phi^{1} \wedge \phi^{3}=\psi^{1} \wedge \psi^{3}, \quad \phi^{2} \wedge \phi^{3}=\psi^{2} \wedge \psi^{3}
$$

it follows that $\epsilon_{1}=\epsilon_{2}=\epsilon_{3}= \pm 1$. Similarly we obtain that

$$
\epsilon_{1}=\cdots=\epsilon_{n}= \pm 1 \text {. }
$$

This completes the proof of Proposition 3.

Theorem 4. Let $M$ be an $n$-dimensional orientable Riemannian space isometrically imbedded into $R^{n+1}$. If the type number $N(u)$ of $M$ at $u$ is greater than or equal to 3 for all $u$ in $P$, then $M$ is rigid in the sense that the isometrical imbedding of $M$ is unique up to a motion in $R^{n+1}$.

Proof. Consider another isometrical imbedding of $M$. Using the same notations as above, we conclude from Proposition 3 that $\phi= \pm \psi$. Choosing a proper spherical map, we may assume that $\phi=\psi$ (see: Remark). Therefore we have

$$
\bar{\xi}^{*}(\bar{\theta})=\bar{\eta}^{*}(\bar{\theta}) .
$$

On the other hand, we have always

$$
\bar{\xi}^{*}(\bar{\omega})=\bar{\eta}^{*}(\bar{\omega}) .
$$

Applying a motion in $R^{n+1}$, we may assume that two imbeddings coincide at $T_{x_{0}}(M)$ for some point $x_{0}$ of $M$. In other words,

$$
\bar{\xi}\left(u_{0}\right)=\bar{\eta}\left(u_{0}\right) \quad \text { for some } u_{0} \text { in } P \text {. }
$$

From Proposition 1 and the fact that $\bar{\theta}$ and $\bar{\omega}$ define an absolute parallelism on $Q$, it follows that $\bar{\xi}=\bar{\eta}$. Now our theorem follows from Proposition 2.

We shall give the geometrical meaning of the type number. Let $\xi: M \rightarrow S$ be the spherical map and let $\delta \xi: T(M) \rightarrow T(S)$ be its differential. Then $N(u)$ $=\operatorname{dim} \delta \xi\left(T_{x}(M)\right)$, where $x$ is the image of $u$ under the projection $\pi$. This can be seen as follows. Let $\bar{u} \in T_{u}(P), \delta \pi(\bar{u})=\bar{x}$ and $\bar{\xi}(u)=u^{\prime}$. Then $\phi$ is given by

$$
\phi(\bar{u})=u^{\prime-1}(\delta \xi(\bar{x})) .
$$

Now our assertion follows immediately.

3. Compact homogeneous hypersurfaces. Let $M$ be a compact $n$-dimensional Riemannian space isometrically imbedded in $R^{n+1}(n \geqq 3)$. Let $d(x)$ be the Euclidean distance between $x \in M$ and the origin $o$ of $R^{n+1}$. Since $M$ is compact and $d$ is a continuous function on $M$, there exists a point $x_{0}$ in $M$ where $d$ attains its maximum. Let $U_{0}$ be a small neighborhood of $x_{0}$ such that $U_{0}$ is 
orientable and the spherical map $\xi: U_{0} \rightarrow S$ is a differentiable homeomorphism of $U_{0}$ onto an open set $\xi\left(U_{0}\right)$ of $S$.

Let $P_{0}$ be the bundle of oriented orthonormal frames over $U_{0}$. Then the type number $N(u)$ is $n$ for all $u$ in $P_{0}$ as we saw at the end of $\S 2$. Since $n \geqq 3$, $U_{0}$ is rigid by Theorem 4 .

Suppose that the group of isometries $I(M)$ of $M$ is transitive on $M$. First of all, we shall show that $M$ is orientable. The tangent space $T_{x_{0}}(M)$ divides $R^{n+1}$ into two parts, say $E_{1}\left(x_{0}\right)$ and $E_{2}\left(x_{0}\right)$. Assume the origin $o$ to be in $E_{1}\left(x_{0}\right)$. Then, in a neighborhood of $x_{0}, M$ is also in $E_{1}\left(x_{0}\right)$. Let $v\left(x_{0}\right)$ be the unit vector normal to $M$ at $x_{0}$ whose end is in $E_{2}\left(x_{0}\right)$. Let $x$ be an arbitrary point of $M$. Since $M$ is homomogeneous, there exists a neighborhood $U$ of $x$ which is isometric to $U_{0}$. Such an isometry is the restriction of a motion in $R^{n+1}$, because $U_{0}$ is rigid. Therefore, a neighborhood of $x$ in $M$ is one side of $T_{x}(M)$, say $E_{1}(x)$. Let $v(x)$ be the unit vector normal to $M$ at $x$ whose end is in the other side $E_{2}(x)$ of $T_{x}(M)$. It is easy to see that the normal vector field $v$, thus chosen, is continuous on $M$. Hence $M$ is orientable.

Since $M$ is orientable, the spherical map $\xi: M \rightarrow S$ is defined in the large. We have seen that $\xi$ is a differentiable homeomorphism of $U_{0}$ onto $\xi\left(U_{0}\right)$. Since $U_{0}$ is rigid and $M$ is homogeneous, $\xi$ is a differentiable homeomorphism in a neighborhood $U$ of every point $x$ of $M$. Therefore $\xi(M)$ is open in $S$. On the other hand, $\xi(M)$ is compact. Therefore $\xi(M)=S$. It can be seen easily that $M$ is a covering space of $S$ with the projection $\xi$. Since $S$ is simply connected, $\xi$ is a differentiable homeomorphism of $M$ onto $S$ in the large.

Since $U_{0}$ is rigid and $M$ is homogeneous, $M$ is rigid. Every isometry of $M$ is, hence, the restriction to $M$ of a motion in $R^{n+1}$. Therefore, the group of isometries $I(M)$ of $M$ is a subgroup of the group of motions in $R^{n+1}$. Since $I(M)$ is compact, it has a fixed point, say $y$, in $R^{n+1}$. Let $x_{1}$ and $x_{2}$ be any points in $M$. There exists an element of $I(M)$ which sends $x_{1}$ into $x_{2}$. Since such a transformation of $M$ is the restriction to $M$ of a motion in $R^{n+1}$ which leaves the point $y$ fixed, the Euclidean distance between $x_{1}$ and $y$ is the same as the distance between $x_{2}$ and $y$. Hence $M$ is a sphere with its center at $y$.

REMARK. In this proof we do not use the fact that the spherical map $\xi: M \rightarrow S$ is a differentiable homeomorphism in the large. However this fact is necessary for other proofs which will be presented later.

4. Compact homogeneous surfaces. So far, we have assumed that $n \geqq 3$. Consider now the case $n=2$. Let $I(M)$ be the group of isometries of $M$ and $I^{0}(M)$ is connected component of the identity. Then

$$
\operatorname{dim} I^{0}(M) \leqq 3 .
$$

Since $I^{0}(M)$ is transitive on $M, \operatorname{dim} I^{0}(M) \geqq 2$. Consider first the case $\operatorname{dim} I^{0}(M)=2$. Since $I^{0}(M)$ is compact, it is necessarily abelian. (Note that there is no simple Lie group of dimension less than 3.) As $I^{0}(M)$ is abelian and effective on $M$, its isotropy subgroup consists of only the identity. Hence $I^{0}(M)$ is simply transitive on $M$ and $M$ is a flat torus, which can not be im- 
bedded into $R^{3}$. Consider finally the case $\operatorname{dim} I^{0}(M)=3$. Then the space $M$ is of constant curvature. Since $M$ is compact and is in $R^{3}$, it has to be a sphere in the large.

5. Alternative proofs. It is known $[3 ; 4 ; 6 ; 10]$ that a compact convex hypersurface $M$ is isometric to a sphere if the sum of principal radii of curvature is constant on $M$. In $\S 3$, we proved that every compact homogeneous hypersurface is convex and rigid. (The convexity follows immediately from the fact that the spherical map is one-to-one). From the rigidity and the homogeneity of $M$, it follows that all the elementary symmetric functions of principal radii of curvature are constant on $M . M$ is hence isometric to a sphere. This reasoning may replace the last part of $\$ 3$.

For $n$ even, we have another proof due to Busemann. The Gaussian curvature is the product of principal radii of curvature if $n$ is even. Since it is intrinsic and $M$ is homogeneous, it is constant on $M$. By the compactness of $M$, it is, moreover, positive. By the Hadamard's Theorem [5], $M$ is convex. The Brunn-Minkowski Theorem [11] tells us that the sphere is the only convex hypersurface with constant Gaussian curvature. This proves our theorem for $n$ even.

\section{BIBLIOGRAPHY}

1. E. Cartan, La déformation des hypersurfaces dans l'espace euclidien réel à $n$ dimensions, Bull. Soc. Math. France vol. 45 (1917) pp. 57-121.

2. S. S. Chern, Lecture notes, Chicago, 1952.

3. - Topics in differential geometry, Princeton, 1951.

4. W. Fenchel and B. Jessen, Mengenfunktionen und konvexe Körper, Det Kgl. Danske Videnskabernes Selskab. Matematisk-Fysiske Meddelelser, XVI vol. 3 (1938).

5. J. Hadamard, Sur certaines propriétés des trajectoires en dynamique, J. Math. Pures Appl. vol. 3 (1897) pp. 331-387.

6. C. C. Hsiung, On differential geometry of hypersurfaces in the large, Trans. Amer. Math. Soc. vol. 81 (1956) pp. 243-252.

7. S. Kobayashi, Le groupe des transformations qui laissent invariant le parallélisme, Colloque de topologie, Strasbourg, 1954.

8. - Espaces a connexions affines et riemanniennes symmétriques, Nagoya Mathematical Journal vol. 9 (1955) pp. 25-37.

9. - - Induced connections and imbedded Riemannian spaces, ibid. vol. 10 (1956) pp. $15-25$.

10. T. Kubota, Ueber die Eibereiche im n-dimensionalen Raume, Science Reports of the Tôhoku Imperial University (1) vol. 14 (1925) pp. 399-402.

11. H. Minkowski, Volumen und Oberflächen, Math. Ann. vol. 57 (1903) pp. 447-495.

12. S. Myers and N. Steenrod, The group of isometries of a Riemannian manifold, Ann. of Math. vol. 40 (1939) pp. 400-416.

13. T. Y. Thomas, Riemannian spaces of class one and their characterization, Acta Math. vol. 67 (1936) pp. 169-211.

Institute for Advanced Study, Princeton, N. J.

UNIVERSITY OF WASHINGTON, Seattle, Wash. 